\title{
Augmented Robotics for Learners: A Case Study on Optics
}

\author{
Wafa Johal ${ }^{1,2}$, Olguta Robu, Amaury Dame ${ }^{1}$, Stephane Magnenat ${ }^{3}$ Francesco Mondada $^{1}$
}

\begin{abstract}
In recent years, robots have been surfing on a trendy wave as standard devices for teaching programming. The tangibility of robotics platforms allows for collaborative and interactive learning. Moreover, with these robot platforms, we also observe the occurrence of a shift of visual attention from the screen (on which the programming is done) to the physical environments (i.e. the robot). In this paper, we describe an experiment aiming at studying the effect of using augmented reality (AR) representations of sensor data in a robotic learning activity. We designed an AR system able to display in real-time the data of the Infra-Red sensors of the Thymio robot. In order to evaluate the impact of AR on the learner's understanding on how these sensors worked, we designed a pedagogical lesson that can run with or without the AR rendering.

Two different age groups of students participated in this between-subject experiment, counting a total of 74 children. The tests were the same for the experimental (AR) and control group (no AR). The exercises differed only through the use of $A R$. Our results show that $A R$ was worth being used for younger groups dealing with difficult concepts. We discuss our findings and propose future works to establish guidelines for designing AR robotic learning sessions.

Augmented Reality, Robotics, Optics, Education.
\end{abstract}

\section{INTRODUCTION}

Robotics is a growing industry, and robots are foreseen to be participate in daily activities in the near future. Policymakers have understood the importance of investing in educational tools to teach computer science and robotics at a young age to promote awareness. Since the LOGO turtle [1] and over the last 40 years, robotic platforms for education have changed a lot: sequentially programmable or event-driven, they also integrate a wide spectrum of sensors, actuators, and interfaces. Besides the robot itself, we observe currently an increasing focus on the design of the learning and pedagogical scenario that make use of robots beyond the fields of programming and robotics. There have been a lot of robotics platform kits and platforms that were designed to teach robotics and programming such as the Lego Mindstorm, Beebot and Thymio [2]. Among all these commercially available robots, we are particularly interested in the use of Thymio to teach programming and robotics.

The Thymio II robot, which in this article will be referred to simply as Thymio, is a desktop differential-drive robot (see Figure 11. Measuring $112 \times 110 \times 55 \mathrm{~mm}$, its shape allows it to be placed on a table in several positions, enabling it to have diverse functions in addition to being a mobile robot. Its white color was chosen in order to give a look that is age-

1 Biorobotics Laboratory (BioRob) EPFL, Switzerland

2 Computer-Human Interaction in Learning and Instruction (CHILI) Laboratory EPFL, Switzerland

${ }^{3}$ Game Technology Center, ETH Zurich

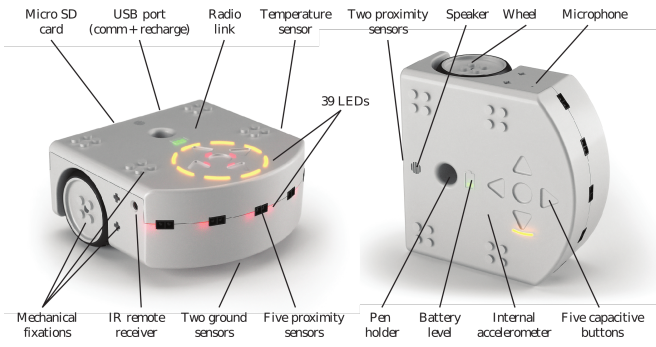

Fig. 1. The Thymio robot and its sensors.

and gender-neutral [3]. Despite its affordable price (around $130 \$$ ), Thymio has a rich set of sensors. A very specific feature of Thymio is the high number of LEDs placed over its body, allowing a visualization of the sensors' activities and creating a high degree of interactivity with the user. At the software level, Thymio is built on the ASEBA framework [4], consisting of a virtual machine running in the robot processor and a very flexible communication infrastructure enabling the programming of the robot and the running of debugging tools over many communication channels.

There are several programming interfaces that can generate code for the on-board ASEBA virtual machine, out of which main ones are:

1) A text-based environment, enabling the use of a simple Matlab-like scripting language.

2) A graphic environment entitled VPL [5], allowing programming for beginners, even children with reading disabilities.

3) Scratch and Blockly environments that use a graphic layout to place text-based code.

The robot also comes with six predefined behavioural modes. One can set the mode by simply selecting it with the top buttons on the robot. Each mode is associated with a color exhibited by the robot. For instance, in the green mode, the robot follows moving objects detected in front of it, while in the red mode, the robot goes back when an obstacle is detected.

In this paper, we explore the use of Augmented Reality (AR) in an educational robotics context to teach several concepts of optics about light spectrum and InfraRed (IR) in a practical session associated to the Physics curriculum.

\section{RELATED WORKS}

In AR, users can see the real world as well as digital objects superimposed over it [6]. In Augmented Virtuality $(\mathrm{AV})$, physical objects are incorporated in a virtual environment. AR and AV are instances of Mixed-Reality that defines a merge of virtual and physical worlds [7]. 
AR has become increasingly accurate, robust, and reliable in the last decades, allowing for the creation of tools that can be targeted at consumer applications. AR enables the users to visualize and to interact with virtual objects or concepts while experiencing an embodied interaction with the real world. This paper focuses on the use of AR although, AV could be relevant for robotics learners as well.

\section{Augmented Reality in Robotics}

Recent research has shown a growing interest in using AR to augment robots [8]. Research has been reported covering several domains of application of the AR technology combined with robotics such as: surgery [9], navigation and teleoperation of remote robots [7], [10] or collocated robots [11], and manufacturing and fabrication [12].

Besides being foreseen to be applicable in several domains, researchers have described what features could AR bring into robotics. For instance, AR could be used to enhance human-robot communication [7]. Autonomous vehicles could show their intentions in terms of path planning, and use on-board intention projection on the floor [13]; bringing a better contextual awareness to the human user with a minimal information sharing. AR can also help users to visualize the robots relative task space (Exo-centric view) [14] or simulate sensor interaction. AR can also be used to enhance collaboration, especially for several people collaborating with a robot [14], [15].

As robotics is being introduced in the school curriculum at earlier and earlier age, benefits for learners who learn about programming with robots have been forseen [16]: (1) by moving, robots attract attention, (2) robots are related to living things and hence attaching, (3) "robots are multidisciplinary systems" that can illustrate STEM sciences (4) "robots have many applications fields".

While these benefits have demonstrated the value of robots in STEM education, they also bring new challenges for learners: (1) attention shifts between the programming interface and the robot that could increase cognitive load; (2) using robots across fields could confuse students in what the robot is supposed to impersonate in the current learning context. These challenges bring new opportunities for AR to help the robot personify entities relevant to the learning context (e.g. a cell in biology or an atom in chemistry) and to reduce attention shift from robot to graphic interface (PC or tablet). In a recent study [17], Magnenat et al. used AR to display in real time the events executed on the robot. Comparing AR with visual feedback, authors found that students in the AR condition made fewer errors, although no significant performance improvement in programming the robot was found. This lesson doesn't aim at teaching programming but rather at introducing concepts of physics; we expect that students with AR feedback would obtain a greater understanding on how the robot's sensors function.

\section{Augmented Reality in K12 Teaching}

AR technology overlays virtual representation (computer graphics) onto real world video stream in real time [18].
Researches using tangible manipulatives combined with AR, suggests that augmented physical objects could be used to support for tangible interface metaphor or transitional interface moving from reality to virtuality [19].

In the past years researchers have investigated several areas that could benefit from AR: assistance, advertisement, navigation, sight-seeing and education [20].

AR has three main features: 1) combining real and virtual content, 2) interactivity in real time, 3) rendered in 3D.

$\mathrm{AR}$ has been used in teaching and learning about phenomena and quantities invisible to naked eye [20], [21]. In a classic setup, to build a 3D intuition of an invisible entity, the teacher would have to play a movie. However, with AR, children can take an active part in the lesson, and can experiment with the graphics in real-time, observing the dynamic properties of the studied phenomenon at their own pace [22]. Hence, AR has been used in STEM fields giving interesting results in maths and physics [23]. Using the AR to generate different view point in a collaborative settings has also been studied [18].

In the current study, we use a commercial device for AR, tablets (Android NVIDIA Gear).

We also aim to discover if the type of concept tackled in the learning activity influences the effect of AR on learning outcomes.

\section{Augmented REAlity FOR Thymio}

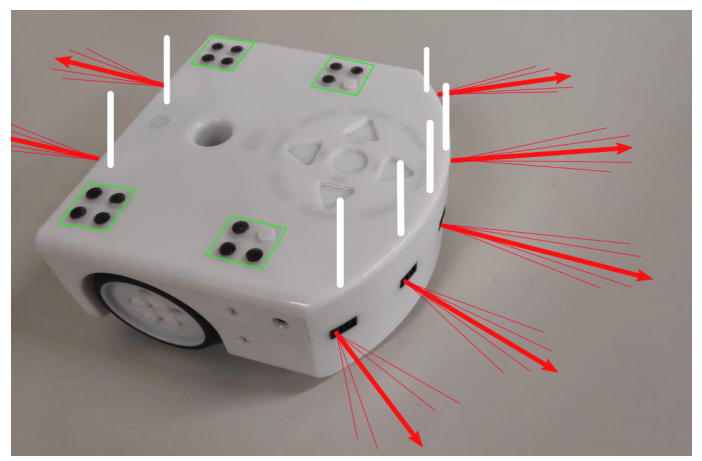

Fig. 2. The red arrows display the direction of the IR emitters, the red lines illustrate the cone range. The white vertical lines show the intensity of the IR signal. In green, we highlight the additional visual pattern that were made on top of the Thymio robot to ease its pose estimation. These green boxes were not shown to the students.

We propose a new framework to augment the Thymio robot and display its sensors' information to the learners. For a device to support AR rendering, it is required to have a camera and display pointing in the opposite direction. There are a number of consumer market devices that allow AR developments: eyeglasses, head mounted displays, tablets or smartphones. We chose to use an Android tablet that can be easily shared among users to display the AR information.

In order to implement the AR activity, and to render the information correctly on the camera image, one needs to determine the 3D position of the tablet's camera relatively to the robot. This comes by first detecting the robot on the camera image, then estimate its position and orientation and 
compute the transformation from the camera to robot frame. Once this transformation is known, the coordinates of the robot in the camera reference frame will allow to overlay and project the sensor's rendering on the robot image.

\section{Detection and Tracking of Thymio}

Knowing the object that needed to be detected, we choose to use a model-based approach for the detection of Thymio. However, Thymio has a glossy look that is subject to specular glare. Besides, its all-white, single shell design makes the segmentation of the robot object from the table background difficult. To compensate for these issues, we textured the robot by coloring in black a few nibs, usually used for attaching Lego bricks onto the robot.

Figure 2 shows how the robot was textured on the top to ease its detection. A first step consists in detecting these black blobs on an image captured by the tablet camera. These blobs are filtered and grouped using their sizes, relative distance and image moment. We then use geometric hashing [24] to match the blob feature with a template. Perspectiven-Point (PnP)[25] is used to determine the 3D pose of the robot.

While such a detection method is fairly robust, it does not run in real-time, it does not take advantage of the time consistency between frames and is therefore likely to give a slightly jittering pose, and finally it only works when the blobs are clearly visible in the image. To overcome those shortcomings, detection is only performed once every ten frames when the tracking is not initialized and, as soon as Thymio is detected, a template-based tracking algorithm is used for the tracking.

Several textured planar parts of Thymio, such as the IR sensors or the side wheels, are tracked in the image by computing the pixels similarity using a Normalized Cross Correlation function, allowing for robustness to light variations. From that we obtained the $2 \mathrm{D}$ pose of those parts in the given image and estimate the corresponding 3D pose of the robot using the PnP approach. We found that this method gave satisfactory results in term of performances $(\approx 30 \mathrm{~Hz}$ and $33 \mathrm{~ms}$ latency).

\section{Display of IR sensors}

In this study, we chose to display with AR, information about the IR sensors. The AR display would show two things overlaid on each IR sensor of Thymio: 1. the cone of IR light coming out of the emitter and displayed with two lines and an arrow. 2. the intensity of the measure captured by the receiver, displayed as a vertical bar on top of the receiver part of the IR sensor. Figure 2 shows the rendering of the information on the tablet app developed for the experiment. As one can see on the image, the IR receiver and emitter were distinct. The arrow coming out only from the emitter side of the sensor and the intensity bar was placed on top of the receiver part of the IR sensor. We also need to mention that the IR sensor was measuring the intensity of the reflected signal and not a distance measure.

\section{THE OPTIC LESSON}

The Thymio optic lesson is a two hours semi-guided session where learners are taught how IR sensors work. The goal of the lesson was to complement some teaching children had on optics with some hand-on session using the Thymio robot.

The session was decomposed in tests, semi-guided practical exercises and challenges. Table II shows details on the learning session design. During the first group activities, learners where guided through a list of exercises that let them experiment with the robot sensors. Each of the exercise sheet presented the goal of the experiment and material needed to run the experiment. For instance, Figure 3, shows how the learner can observe the detection range of the sensors using a small piece of white paper.

\begin{tabular}{lrll}
\hline Step & Duration & Activity & Org. \\
\hline 1 & $10 \mathrm{~min}$ & Presentation of Thymio & Class \\
2 & $10 \mathrm{~min}$ & Pretest & Ind \\
3 & $5 \mathrm{~min}$ & Making the groups & Grp \\
4 & $10 \mathrm{~min}$ & Semi-Guided Exploration & Grp \\
5 & $10 \mathrm{~min}$ & Midtest & Ind \\
6 & $10 \mathrm{~min}$ & Practical Exercises & Grp \\
7 & $10 \mathrm{~min}$ & Challenges & Grp \\
8 & $10 \mathrm{~min}$ & Posttest & Ind \\
\hline
\end{tabular}

TABLE I

SESSION Flow With IND.: INDIVIDUAL ACTIVITIES, GPR.: GROUP ACTIVITIES

While in the Semi-Guided exploration activity, students could explore with the system, in the practical exercises, learners were asked to perform specific tasks and to answer questions, such as determining the detection range of the sensors by taking measurements on a grid paper placed under the robot. Here again, the material required and procedure were described for students to perform the task. Finally,

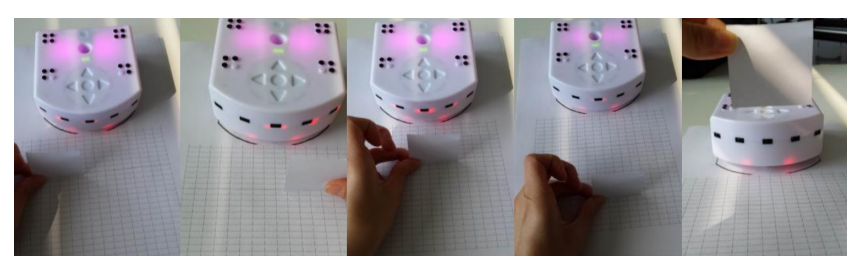

Fig. 3. Example of exercise in the semi-guided exploration activity for which student explore the intensity of the IR signal reflected according to the distance of the sensor with regard to a white piece of paper.

for the challenges, students could openly use any of the available material to solve the tasks. The teachers proposed 4 challenges that required students to have understood the previously taught concepts. For instance, one of the challenges was to touch the robot's front face without triggering the IR sensors. This could be achieved by understanding the conical shape of the IR beam and finding the resulting blind spot. 


\section{Four concepts of optics}

The whole session was built to make children discover about the IR sensors working principles. Children used the IR sensors of the Thymio robot to experiment and test their hypothesis in a guided discovery session. Below the list of target concepts of the learning activity:

C1 : IR are composed of an emitter and a receiver: an IR emitting diode and a silicon phototransistor.

C2 : The emitter emits an IR beam of conical shape.

C3 : The measure is made in IR: it is invisible. The receiver measures the amount of received IR light of a certain range of wavelengths.

C4 : The measure is based on reflection. The amount of IR light an obstacle reflects back to the emitter depends on: the distance of the obstacle the emitted beam meets, its position, the texture/material and its color.

These concepts are inline with actual school curriculum in optics.

\section{Tests}

Students took a pretest, a midtest and a posttest to check their understanding of the four concepts that were targeted with our activity (see Table II). These tests consisted of three different sets of multiple choice questions with one or several correct answers. The tests were growing in difficulty and length (4, 8 and finally 10 questions). Students answered each of the tests individually. Each question would be linked with one or two concepts. The grading was consisting in giving a point if the answer provided by the student showed that (s)he understood a concept. Some items could validate the understanding of one concept but be wrong for another one. For instance:

What makes the obstacle sensors of Thymio?

a) An emitter and receiver of invisible light.

b) An emitter and receiver of visible light.

c) Several emitters and receivers of invisible light.

d) An emitter, a receiver and a LED.

e) A red laser.

In this example of question, two concepts (C1 and $\mathrm{C} 3)$ are covered.

\section{EXPERIMENTAL SETUP}

\section{Participants and Methods}

74 students from two classrooms participated in this study from two schools of Switzerland and France. None of the students had previous experience with the Thymio robot. We recruited students from two age-groups. All students participated with parental or guardian consents. In the group of younger children (M: 10 yo, SD: 0.8, with 26 girls), there were 45 students grouped in pairs for the learning sessions (tests were taken individually). The second group of 29 students were older (M: 15 yo, SD: 0.8, with 10 girls). Students from this older team were also paired for the learning activities.

For the two age groups, pairs of students were distributed in the AR or NOAR conditions. The two groups performed the tasks in two different rooms but had the same equipment and material apart from the AR tablet for the students in the AR condition. The AR and NOAR group were formed splitting students in order to form similar groups in term of science and math grades. The teacher giving the AR and NOAR lessons also swapped between the sessions.

In the AR condition, students could visualize the IR intensity via the app as described in Section III In the NOAR condition, we used the intensity of the robot's LEDs to reflect the IR intensity.

\section{Hypothesis}

H1 Students in the Augmented Reality condition will obtain greater learning gains than students in the NOAR condition.

$\mathrm{H} 2$ Is there a different impact of $\mathrm{AR}$ on the learning gain depending on the types of concept influences the impact of AR on learning gain.

H3 The learning impact of Augmented Reality information depends on the age level. We expect younger children to benefit more from the AR representations than the elder students.

\section{RESUlTS}

In order to assess the results of our experiment we used the Mann-Whitney $U$ test as we have a between-subject experimental design and the test scores are not normally distributed.

The main results that we present here concern the individual tests. During the challenges, the excitement of the class made it difficult to record valuable extra data such as time to solve a task or value of the proposed method.

As described in Section V, the pretest, midtest and posttest consisted of multiple choice questions with multiple answer possible. The grading was done in such a way that the student would get the full mark for the given question, if (s)he selected all the correct items. If items were missing or incorrectly selected, the score was decreased.

\section{Overall Learning Gain}

Figure 4 shows the mean score of students for the AR and NOAR groups at each test. Both group show a significant increase of learning gain during the activity, validating the fact that the pedagogical scenario was well designed. The groups in the AR condition seem to slightly outperform the NOAR groups. This difference is significant for the posttest, and close to significant for the pretest $(p=0.082)$. However, the learning gain, computed as the difference between the post and pretest, is not significantly different between these two conditionss. This could be due to an unbalanced group split of students between conditions even if groups were meant $o$ be balanced and made by the teachers based on student's sciences grades.

\section{Influence of $A R$ according to the concept}

In order to assess if the AR condition was influencing differently the learning according to the concept taught, we run a statistical analysis for each concept on the individual 


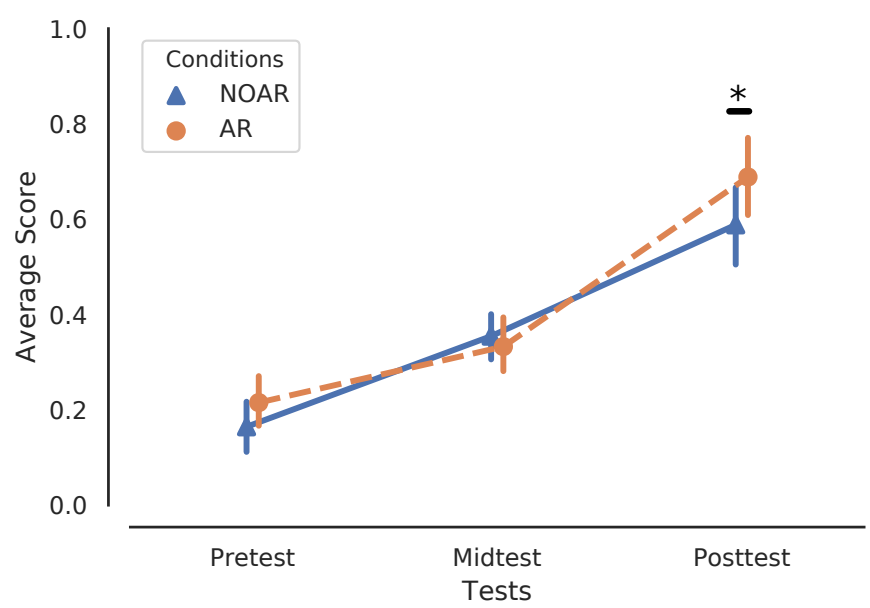

Fig. 4. Average score for the AR and NOAR groups for each test. Statistically significant higher score was observed between the AR and NOAR group for the posttest $(U(36,38)=517, p=0.03)$

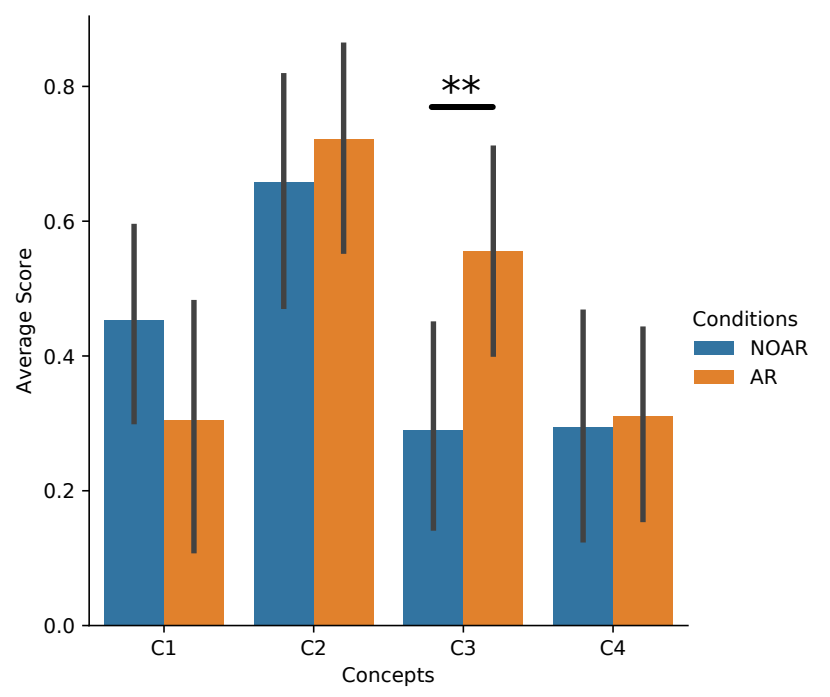

Fig. 5. Average individual learning gain for each concept and each condition. Statistically significant higher learning gain was observed between the $\mathrm{AR}$ and NOAR group for the Concept $3(U(36,38)=479, p=0.009)$

learning gains. Figure 5 shows the average of individual learning gains for each concept and AR conditions. We can see a statistically significant difference between AR and NOAR only for the Concept 3 . It is interesting to notice that the Concept 3 deals with the fact that IR light is invisible. A plausible explanation is that children could shift from using the AR tablet or not in the AR condition. That made it obvious that what they could see through the tablet was actually invisible to the naked eye.

\section{Influence of $A R$ according to the age}

It is interesting to study the influence of age range of the students on the impact of AR. Here, we wondered if AR impact differently students both positively of negatively. Figure 6 presents the average learning gain for Youngs and Older groups in the two conditions AR and NOAR. No interaction effect was found, but a statistical difference was

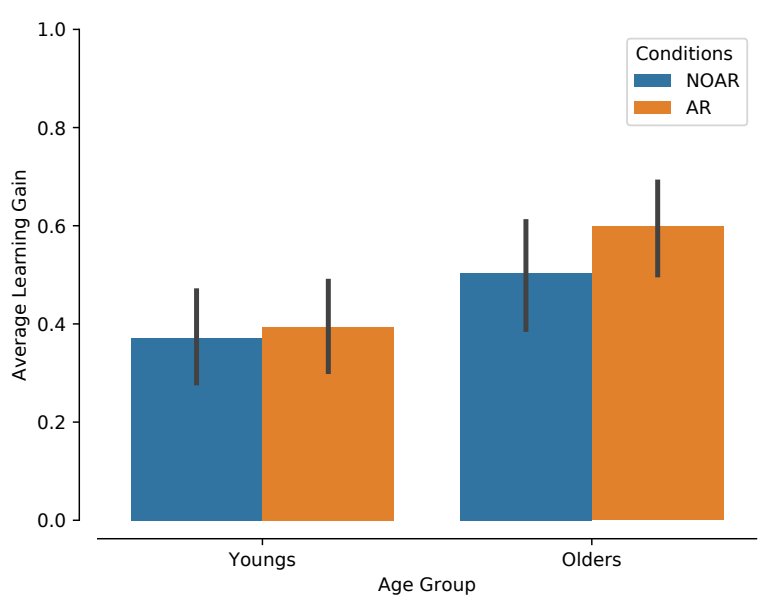

Fig. 6. Average individual learning gain the two age group and each condition.

found between the young and elder group $(p<0.023)$. This might be explained by the fact that the topic was introduced similarly to the two groups and that the content of the lesson was better adapted to the elder age group.

\section{DISCUSSION AND FUTURE WORK}

There are a number of considerations with regards to the generalizability of our study results. First, the IR cone was actually not rendered with a cone but with an arrow and lines. These was due to technical implementation issues within the AR application. We are currently investigating other rendering techniques that could allow to render $3 \mathrm{D}$ objects over the 2D image, allowing the use of cones to visualize the beam. The AR Core API is a promising candidate for this purpose.

In addition, it can be interesting to test in the future other types of rendering for the IR Beam and intensity. Other work have shown that the rendering of the AR could be both beneficial or undesirable depending on the context [18]. Thus, designer will need to take this into account in order to take advantages of certain types of rendering for better comprehension.

Breaking down the effect of AR on the different concepts tackled in this experiment, we found that the concept $\mathrm{C} 3$, tiding with making the invisible visible benefited the most of AR. These results support research indicating that AR is suitable to make the invisible visible [20].

For now, interaction is just by moving around the object - using only the IMU and camera sensor of the tablet. Touch screens could be made interactive, i.e display in tooltip additional information such as raw numbers from the sensors, give hints and questions about reaching a goal within the learning activity.

Because of the system setup, it is hard to monitor and assess the cognitive process of children with AR compared to NOAR conditions. Indeed, understanding the in-task use of the AR related to the performance of the learning teams 
could give us good insight of the reason why AR could be beneficial. Future work integrating behavioral analysis should be conducted by analyzing manipulation and activity logs (combining metrics such as Tablet IMU, robots status, activity click stream).

Finally, the example of this scenario shown in this paper is limited to one type of Thymio's sensor. In the future, we would like to study the use of Augmented Reality in Robotics Education for other types of informative visual feedback, such as motor status. This could help learners in programming to debug their implementation more easily.

\section{CONCLUSION}

In this paper, we investigated how AR could provide richer information to learners. To do so, we introduced a new type of robotics AR system that can render sensor status of robots. We described the implementation of the robot pose estimation from visual markers and demonstrated its possibilities with a learning scenario in Optics. We evaluated how students can benefit from it in a rigorously-designed learning activity combining the Thymio robots and AR, the subject of which was selected from the actual school curriculum. We presented the lesson design with its didactic sequence to let the learners explore, apply and transfer the knowledge about optical properties of IR sensors of the Thymio robot. We hope that this paper and our study will spur interests and aid researchers in further exploring the use of AR combined with robots in education.

\section{ACKNOWLEDGMENTS}

We thank participants and teachers for their cooperation during this study, and members of the CVLab at EPFL who helped in implementing the AR system. We would also like to thank the Swiss National Science Foundation for supporting this project through the National Centre of Competence in Research Robotics as well as the Swiss CTI Grant 17479.2 pfes-es.

\section{REFERENCES}

[1] M. Resnick, S. Ocko, and S. Papert, "Lego, logo, and design," Children's Environments Quarterly, pp. 14-18, 1988.

[2] M. E. Karim, S. Lemaignan, and F. Mondada, "A review: Can robots reshape k-12 STEM education?," in 2015 IEEE International Workshop on Advanced Robotics and its Social Impacts (ARSO), pp. 1-8, IEEE, 2015.

[3] F. Riedo, M. Chevalier, S. Magnenat, and F. Mondada, "Thymio ii, a robot that grows wiser with children," in 2013 IEEE Workshop on Advanced Robotics and its Social Impacts, pp. 187-193, Nov 2013.

[4] S. Magnenat, P. Rtornaz, M. Bonani, V. Longchamp, and F. Mondada, "Aseba: A modular architecture for event-based control of complex robots," IEEE/ASME Transactions on Mechatronics, vol. 16, pp. 321329, April 2011.

[5] J. Shin, R. Siegwart, and S. Magnenat, "Visual programming language for Thymio II robot," in Proc. of the 2014 Conference on Interaction Design and Children (IDC), 2014.

[6] R. Azuma, Y. Baillot, R. Behringer, S. Feiner, S. Julier, and B. MacIntyre, "Recent advances in augmented reality," IEEE Computer Graphics and Applications, vol. 21, pp. 34-47, Nov 2001.

[7] P. Milgram, S. Zhai, D. Drascic, and J. Grodski, "Applications of augmented reality for human-robot communication," in Proceedings of 1993 IEEE/RSJ International Conference on Intelligent Robots and Systems (IROS'93), vol. 3, pp. 1467-1472, IEEE, 1993.
[8] W. Hoenig, C. Milanes, L. Scaria, T. Phan, M. Bolas, and N. Ayanian, "Mixed reality for robotics," in 2015 IEEE/RSJ International Conference on Intelligent Robots and Systems (IROS), pp. 5382-5387, IEEE, 2015.

[9] P. Pessaux, M. Diana, L. Soler, T. Piardi, D. Mutter, and J. Marescaux, "Towards cybernetic surgery: robotic and augmented reality-assisted liver segmentectomy," Langenbeck's Archives of Surgery, vol. 400, pp. 381-385, Apr 2015.

[10] R. Marin, P. J. Sanz, and J. S. Sánchez, "A very high level interface to teleoperate a robot via web including augmented reality," in Proceedings 2002 IEEE International Conference on Robotics and Automation (Cat. No. 02CH37292), vol. 3, pp. 2725-2730, IEEE, 2002.

[11] H. Hedayati, M. Walker, and D. Szafir, "Improving collocated robot teleoperation with augmented reality," in Proceedings of the 2018 ACM/IEEE International Conference on Human-Robot Interaction, pp. 78-86, ACM, 2018.

[12] G. Michalos, P. Karagiannis, S. Makris, nder Tokalar, and G. Chryssolouris, "Augmented reality (ar) applications for supporting humanrobot interactive cooperation," Procedia CIRP, vol. 41, pp. $370-$ 375, 2016. Research and Innovation in Manufacturing: Key Enabling Technologies for the Factories of the Future - Proceedings of the 48th CIRP Conference on Manufacturing Systems.

[13] R. T. Chadalavada, H. Andreasson, R. Krug, and A. J. Lilienthal, "That's on my mind! robot to human intention communication through on-board projection on shared floor space," in 2015 European Conference on Mobile Robots (ECMR), pp. 1-6, Sep. 2015.

[14] S. A. Green, M. Billinghurst, X. Chen, and J. G. Chase, "Human-robot collaboration: A literature review and augmented reality approach in design," International Journal of Advanced Robotic Systems, vol. 5, no. 1 , p. 1, 2008.

[15] M. Billinghurst, H. Kato, and I. Poupyrev, "Collaboration with tangible augmented reality interfaces," in HCI international, vol. 1, pp. 5-10, 2001.

[16] F. Riedo, P. Rétornaz, L. Bergeron, N. Nyffeler, and F. Mondada, "A two years informal learning experience using the thymio robot," in Advances in Autonomous Mini Robots, pp. 37-48, Springer, 2012.

[17] S. Magnenat, M. Ben-Ari, S. Klinger, and R. W. Sumner, "Enhancing robot programming with visual feedback and augmented reality," in Proc. of the 2015 ACM Conference on Innovation and Technology in Computer Science Education (ITiCSE), pp. 153-158, ACM, 2015.

[18] H.-K. Wu, S. W.-Y. Lee, H.-Y. Chang, and J.-C. Liang, "Current status, opportunities and challenges of augmented reality in education," Computers Education, vol. 62, pp. 41 - 49, 2013.

[19] M. Billinghurst, H. Kato, and I. Poupyrev, "Tangible augmented reality," ACM SIGGRAPH ASIA, vol. 7, 2008.

[20] M.-B. Ibez and C. Delgado-Kloos, "Augmented reality for STEM learning: A systematic review," vol. 123, pp. 109 - 123, 2018.

[21] M. Alrashidi, K. Almohammadi, M. Gardner, and V. Callaghan, "Making the invisible visible: Real-time feedback for embedded computing learning activity using pedagogical virtual machine with augmented reality," in Augmented Reality, Virtual Reality, and Computer Graphics (L. T. De Paolis, P. Bourdot, and A. Mongelli, eds.), (Cham), pp. 339355, Springer International Publishing, 2017.

[22] M. Akçayır, G. Akçayır, H. M. Pektaş, and M. A. Ocak, "Augmented reality in science laboratories: The effects of augmented reality on university students laboratory skills and attitudes toward science laboratories," Computers in Human Behavior, vol. 57, pp. 334-342, 2016.

[23] J. Bacca, S. Baldiris, R. Fabregat, S. Graf, et al., "Augmented reality trends in education: a systematic review of research and applications," 2014.

[24] H. J. Wolfson and I. Rigoutsos, "Geometric hashing: An overview," IEEE computational science and engineering, vol. 4, no. 4, pp. 10-21, 1997.

[25] L. Quan and Z. Lan, "Linear n-point camera pose determination," IEEE Transactions on pattern analysis and machine intelligence, vol. 21, no. 8, pp. 774-780, 1999. 